\title{
Pulsed Power and Power Conditioning Capacitors
}

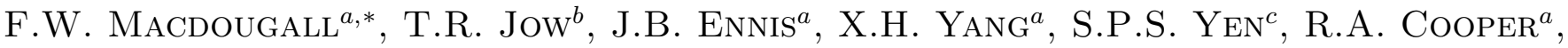 \\ J.E. Gilbert ${ }^{a}$, M. Schneider $^{a}$, C. NARUo ${ }^{a}$ AND J. BAteS ${ }^{a}$ \\ ${ }^{a}$ General Atomics Electronic Systems, Inc., San Diego, CA, USA \\ ${ }^{b}$ US Army Research Laboratory, Adelphi, MD, USA \\ ${ }^{c}$ NASA Jet Propulsion Laboratory, Pasadena, CA, USA
}

\begin{abstract}
A capacitor development effort focused on capacitors used in pulsed power and power conversion applications underwent at General Atomics Electronic Systems Inc. (GA-ESI) for decades. In recent years, funding for these efforts has accelerated the rate of development progress to the point where the time it takes to decrease the size of a capacitor by half has dropped from 10 years to 4 years. This progress made in pulsed power and power conversion capacitors will be described along with the performance characteristics of today's capacitors.
\end{abstract}

PACS numbers: 84.32.Tt, 84.60.Ve

\section{Introduction}

Today's mobile platforms in both the military and commercial sectors, have resulted in a demand for higher performance capacitors [1-3]. A primary concern is energy density, and in the areas where funding has been available, the energy density of the capacitors has been doubling about every four years. This has increased interest in what might be achieved if research continues in these areas as well as other areas. One of the new areas of interest are high-temperature power conversion capacitors. Presently mobile platforms are using separate lower temperature cooling systems to ensure the temperature of electronic components below their maximum operation temperature. The capacitor is one of the components that needs to be cooled at a relatively low temperature and improvements in the capacitors temperature performance would be instrumental in the elimination of a special electronic power conditioning cooling system.

\section{Millisecond pulse width discharge capacitors}

For the last two decades, the energy density of film dielectric capacitors designed to deliver energy in the millisecond range has steadily improved. This has resulted from better dielectric materials, better construction techniques, and perhaps most importantly, the use of self-healing metallized electrodes. The capacitors using these electrodes survive thousands of dielectric breakdowns with the only permanent effect being a very small loss in capacitance. The effect of a single dielectric breakdown and clearing event is immeasurable in a large capacitor but thousands of breakdowns result in a measurable

\footnotetext{
* corresponding author; e-mail: fred.macdougall@ga-esi.com
}

loss of capacitance. For the most part, $5 \%$ capacitance loss is used to describe the end of life for a capacitor although the capacitor can continue to perform function when $5 \%$ capacitance loss is reached.
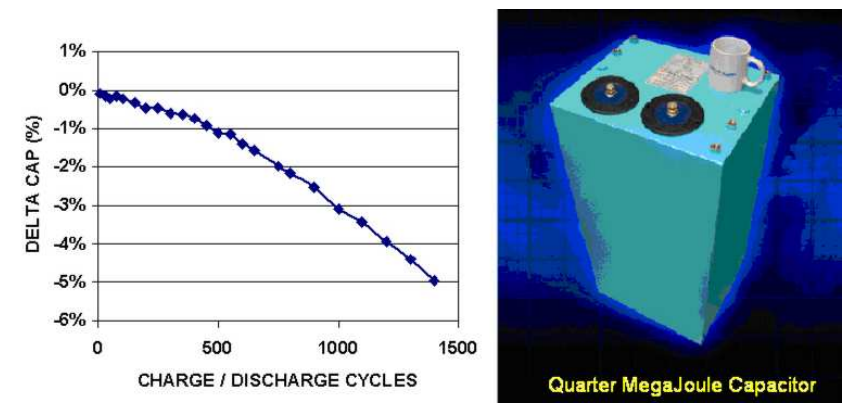

Fig. 1. Life expectancy of a typical quarter megajoule capacitor designed to discharge with a millisecond pulse width.

The softer failure mode associated with self-healing has lead to higher energy density capacitors, higher reliability, and larger capacitors. In the past few years, capacitors like that shown in Fig. 1 have become available with more than $250000 \mathrm{~J}$ stored in one can. In Table life expectancy of millisecond pulse width energy discharge capacitors is shown.

\section{Microsecond pulse width discharge capacitors}

In the past few years, energy storage capacitors designed to discharge in the microsecond time frame using metallized electrodes, have become available. The microsecond pulse width discharge capacitors exhibit the same soft failure mode as the slower millisecond pulse 
TABLE

Life Expectancy of Millisecond Pulse Width Energy Discharge Capacitors

\begin{tabular}{c|c}
\hline \hline $\begin{array}{l}\text { Life Expectancy } \\
\text { to 5\% Cap Loss }\end{array}$ & $\begin{array}{c}\text { Volumetric Energy } \\
\text { Density }\end{array}$ \\
\hline 1000 & $3 \mathrm{~J} / \mathrm{cc}$ \\
10000 & $2.5 \mathrm{~J} / \mathrm{cc}$
\end{tabular}

width discharge capacitors but at lower energy densities. General Atomic Electronic Systems Inc. (GA-ESI) has achieved over $200 \mathrm{~V} / \mu \mathrm{s}$ at $10 \mathrm{kV}$ in high energy density microsecond discharge self-healing capacitors. The faster rise time results in higher current concentration in the capacitor. In terms of volumetric energy density, the faster microsecond capacitors operate at about $70 \%$ of the density of an equivalent millisecond discharge capacitor. The actual performance is dependent on the specific applications.

The application requirements for microsecond discharge capacitors often include a duty cycle with significant DC life expectancy. In terms of DC life expectancy, these capacitors can hold a charge for 2000 hours when operating at $1.3 \mathrm{~J} / \mathrm{cc}$. Figure 2 shows typical test data from a high energy density microsecond discharge capacitor under DC life test conditions.

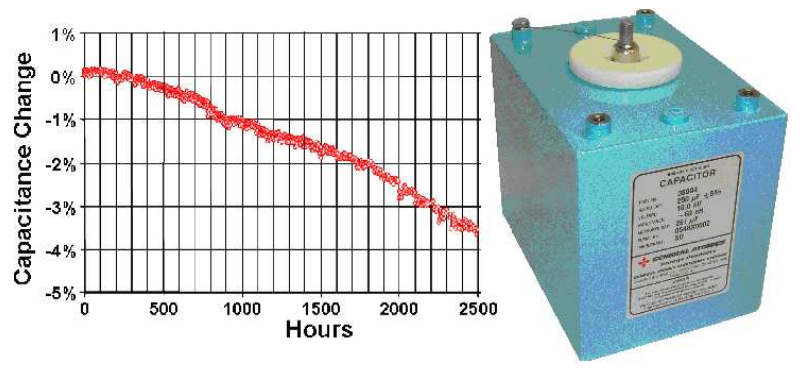

Fig. 2. DC life test data at $1.3 \mathrm{~J} / \mathrm{cc}$ for a typical microsecond pulse width discharge capacitor.

\section{Sub-microsecond pulse width discharge capacitors}

GA-ESI designs and manufactures capacitors that operate in pulse discharge applications where the pulse width is far less than a microsecond. High currents make it impractical to use metallized electrodes. Utilizing solid aluminium electrodes, rise time in the order of $0.2 \mathrm{MV} / \mu \mathrm{s}$ can be achieved. The capacitors are not self healing and unlike their metallized electrode counterpart, these capacitors will fail completely on the first dielectric breakdown.

Figure 3 shows typical sub-microsecond pulse discharge capacitors. The capacitor in the outline drawing on the left and in the left side of the photograph is an aluminium
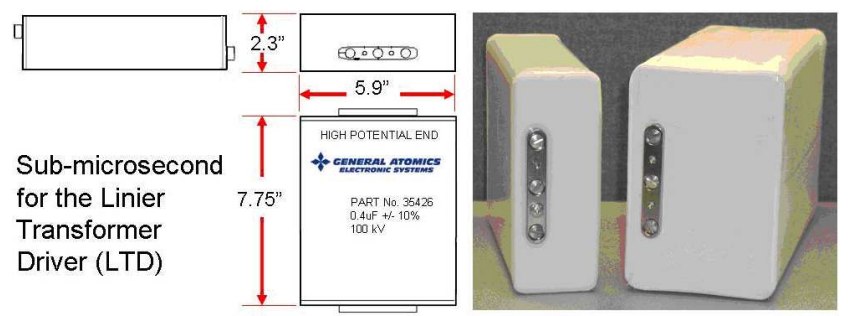

Fig. 3. Typical sub-microsecond pulse width discharge capacitor.

foil electrode capacitor delivering $2 \mathrm{~kJ}$ at $100 \mathrm{kV}$ with an energy density of $1.2 \mathrm{~J} / \mathrm{cc}$. The capacitor on the right in the photo is a similar $10 \mathrm{~kJ}$ capacitor. Both capacitors are designed to for Linier Transformer Drivers (LTDs). This type of capacitors has a self resonant frequency as high as $7 \mathrm{MHz}$.

\section{Power conversion capacitors}

Power conversion capacitors have requirements that include high RMS currents that often drive the design. The heating of the capacitor is due to both the surrounding equipment and the heat generated internally. The internal heat generated can be minimized by increasing the conductivity of the electrodes and by making the active width (A.W.) of the electrode shorter. The solid aluminium electrodes have a higher conductivity and generate less heat than metallized electrodes. Shorter active widths lower the current concentration in the electrode resulting in lower $I^{2} R$ losses. Figure $4 \mathrm{a}$ is a typical di-

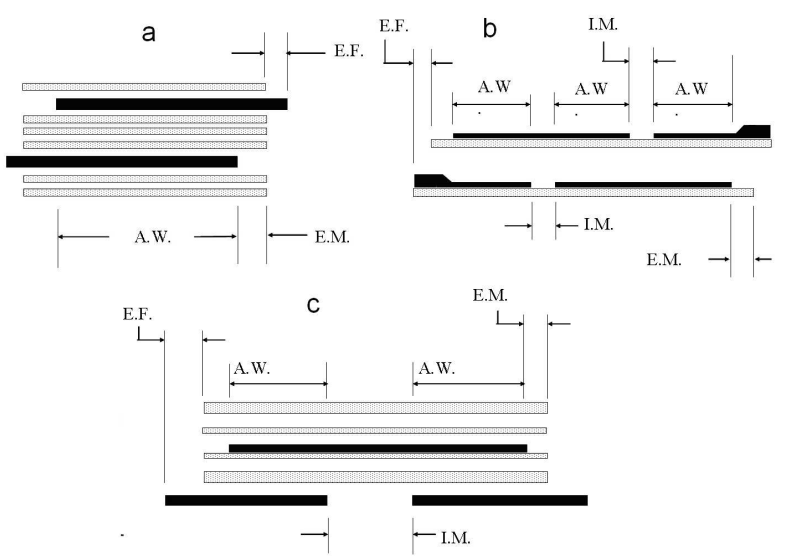

Fig. 4. Cross section of typical power conversion capacitor.

electric and electrode arrangement using aluminium foil electrodes, and extended foil (E.F.) on both sides and an end margin (E.M.) on both ends. Figure $4 \mathrm{~b}$ shows using metallized electrodes with shorter active widths and inner-electrode margins (I.M.) to reduce the internal heat generation. Figure 4c shows using aluminium foil for the 
outside electrodes and a metallized electrode for the foils buried in the capacitor winding.

\section{Conclusions}

The performance of pulse power and power conversion capacitors continues to improve. With the present level of research, the capacitors are expected to double in energy density every 4 years. At the present activity level, this trend is expected to continue for the foreseeable future.

\section{Acknowledgments}

Portions of the research reported in this document/presentation were performed in connection with contract W911QX-04-D-0003 with the U.S. Army Research Laboratory. The views and conclusions contained in this document/presentation are those of the authors and should not be interpreted as presenting the official policies or position, either expressed or implied, of the
U.S. Army Research Laboratory or the U.S. Government unless so designated by other authorized documents. Citation of manufacturers' or trade names does not constitute an official endorsement or approval of the use thereof. The U.S. Government is authorized to reproduce and distribute reprints for Government purposes notwithstanding any copyright notation hereon.

\section{References}

[1] J.B. Ennis, F.W. MacDougall, X.H. Yang, R.A. Cooper, K. Seal, C. Naruo, B. Spinks, P. Kroessler, J. Bates, IEEE Pulsed Power and Plasma Science Conference, Albuquerque, New Mexico 2007, p. 265.

[2] F. MacDougall, T.R. Jow, J. Ennis, S.P.S. Yen, X.H. Yang, J. Ho, IEEE Power Modulator Conference, and High Voltage Conference, Las Vegas 2008. Proceedings of 2008, p. 167.

[3] J.B. Ennis, F.W. MacDougall, X.H. Yang, A.H. Bushnell, R.A. Cooper, J.E. Gilbert, IEEE Power Modulator Conference and High Voltage Conference, Las Vegas 2008, p. 53. 